\title{
flbD encodes a Myb-like DNA-binding protein that coordinates initiation of Aspergillus nidulans conidiophore development
}

\author{
Jenny Wieser and Thomas H. Adams \\ Department of Biology, Texas A\&M University, College Station, Texas 77843 USA
}

\begin{abstract}
The timing of asexual fruiting body formation during Aspergillus nidulans colony development is precisely regulated so that conidiophores are typically produced 1-2 $\mathrm{mm}$ behind the growing edge of the colony. Mutations in any of four $A$. nidulans genes, $f l b B, f l b C, f l b D$, or $f l b E$, result in colonies that are delayed at least $24 \mathrm{hr}$ in their ability to initiate conidiophore development resulting in fluffy colonies with conidiophores forming in the center, at least 12-15 mm behind the growing edge. The requirement for each of these four genes in determining the timing of developmental initiation precedes transcriptional activation of the primary developmental regulatory gene $b r l A$, indicating a possible role for each gene in developmentally regulated activation of brlA expression. The wild-type flbD gene was isolated and shown to encode an $\sim 1.6-\mathrm{kb}$ mRNA that is present throughout the $A$. nidulans life cycle. The deduced FlbD protein sequence predicts a 314-amino-acid polypeptide with significant identity at its amino terminus to the DNA-binding domain of the Myb family of transcription factors indicating that FlbD probably functions as a sequence-specific transcriptional activator. Although conidiophore development does not normally occur in submerged culture, forced overexpression of $f I b D$ in submerged hyphae caused inappropriate activation of brlA expression and resulted in production of complex conidiophores that produced all of the distinct cell types observed in wild-type conidiophores including viable spores. This ability of $f l b D$ overexpression to activate conidiation requires brlA, $f l b B$, and $f l b A$ (another early developmental regulator) but does not require $f l b C$ or $f l b E$. We propose that FlbD functions during normal development by activating transcription of other genes required for development (such as brlA) and that FlbD activity is normally controlled post-transcriptionally by an unknown mechanism.
\end{abstract}

[Key Words: $f l b D$; myb; conidiation; fungi; brlA; microbial development]

Received December 7, 1994; revised version accepted January 20, 1995

Asexual reproduction in the filamentous fungus $A s$ pergillus nidulans is a continual progression that begins with newly forming hyphae at the growing edge of the colony and progresses through differentiation of specialized cells $1-2 \mathrm{~mm}$ behind the leading edge of the mycelium and gives rise to multicellular spore-bearing structures, termed conidiophores (Navarro-Bordonaba and Adams 1994). The initiation of $A$. nidulans conidiophore development, in contrast to initiation of sporulation in many other microorganisms, is not normally triggered by unfavorable environmental conditions but instead occurs as a precisely timed, genetically programmed event in the life cycle in response to internal and external cues (Pastushok and Axelrod 1976; Champe et al. 1981; Adams et al. 1992). The molecular genetic events regulating onset of conidiation are not fully understood but ultimately result in the controlled activation of a transcriptional cascade leading to the expression of hundreds of essential /Clutterbuck 1969; Marti- nelli and Clutterbuck 1971; Stringer et al. 1991) and nonessential genes (Aramayo et al. 1989). Several lines of evidence indicate that a primary activator of sporulation-specific gene expression is BrlA, a C2H2 zinc finger nucleic acid-binding protein (Adams et al. 1988, 1990; Chang and Timberlake 1992). brlA mRNA is present at extremely low levels in hyphae and begins to accumulate shortly after developmental induction (Boylan et al. 1987; Han et al. 1993; Prade and Timberlake 1993). brlA is required for activation of other sporulation-specific genes including at least two developmental regulatory genes, $a b a A$ and wet $A$ while $b r l A$ null mutants produce abnormal developmental structures that initially resemble conidiophore stalks but grow indeterminately, failing to differentiate other specialized cell types (Clutterbuck 1969; Boylan et al. 1987). The pivotal role for $b r l A$ in controlling activation of the conidiation pathway is most strongly supported by the observation that forced activation of brlA in vegetative hyphae re- 
sults in misscheduled activation of other genes, including $a b a A$ and wet $A$, and causes hyphal tips to differentiate into reduced conidiophores that produce viable conidia (Adams et al. 1988). These results focus the question of what activates conidiation on what induces brlA expression.

We have identified and begun to characterize six separate loci, designated $f l u G, f l b A, f l b B, f l b C, f l b D$, and $f l b E$, that are independently required for normal activation of brlA expression (Wieser et al. 1994). Mutations in any one of these genes result in colonies that fail to make the programmed switch from undifferentiated vegetative hyphal growth at the edge of the colony to conidiophore development within. These mutants have diverse phenotypes, but all produce large masses of undifferentiated aerial hyphae that give the colony a "fluffy" or cottonlike appearance. Fluffy mutants resulting from loss of fluG function can be distinguished from strains with mutations in any of the other five genes in at least two ways. First, although fluG mutants are unable to activate brlA expression normally, the requirement for flu $G$ can be partially overcome by limiting growth nutritionally (Adams et al. 1992). Second, the fluG mutant phenotype is suppressed when fluG mutants are grown next to wildtype colonies, even if the two colonies are separated by a dialysis membrane with a 6000 - to 8000 -dalton pore size (Lee and Adams 1994a). These results led us to propose that fluG is responsible for the synthesis of a low-molecular-weight factor that functions as an extracellular signal in activating the major programmed sporulation pathway. In the absence of $f l u G$, however, development can be activated by a mechanism that involves sensing growth rate or nutrient status directly, bypassing the need for FluG.

Mutations in the $1 b A$ gene result in colonies that have abnormal hyphal morphology as well as being altered in their ability to produce conidiophores /Lee and Adams 1994b). The hyphae found at the leading edge of flbA mutant colonies grow close together in tight clumps rather than in a well-separated branching pattern as is observed for wild-type and other fluffy mutant colonies. In addition, flbA mutant colonies produce tightly interwoven aerial hyphae that autolyse beginning in the center of the colony so that by 5 days postinoculation the entire colony has disintegrated. The predicted $f l b A$ product is related to the Saccharomyces cerevisiae SST2 gene product that has been implicated in regulating the activity of the G-protein-mediated signal transduction pathway that controls yeast mating (Dietzel and Kurjan 1987). The similarity observed between $f 1 b A$ and SST2 has led to the proposal that FlbA could function to regulate the activity of a signal transduction pathway for responding to the putative fluG signal for conidiation (Lee and Adams 1994a,b). Although this hypothesis has not been tested directly, we have shown that $f l b A$, like brlA, does have a direct role in activating development because overexpression of $f l b A$ in submerged culture is sufficient to cause activation of $b r l A$ expression and the production of viable spores (Lee and Adams 1994b).

Mutations in $f l b B, f l b C, f l b D$, or $f 1 b E$ result in a third, distinct class of fluffy mutant colony described as delayed conidiation (Wieser et al. 1994). This phenotype differs from the fluffy phenotype observed for fluG and flbA mutants in that delayed conidiation mutant colonies always produce conidiophores in the center after extended incubation and the colony margin remains fluffy. In this paper we describe the isolation and initial characterization of one delayed conidiation gene, $f l b D$. The $f l b D$ gene is predicted to encode a 314-amino-acid polypeptide with significant identity in its amino terminus to the DNA-binding domain found in a group of transcription factors that includes the human proto-oncogene c-myb (Majello et al. 1986; Lüscher and Eisenman 1990). Forced overexpression of $f 1 b D$ in submerged culture leads to the production of complete conidiophores that include stalks, vesicles, and sterigmata that produce viable spores. This contrasts with development observed following forced expression of $b r l A$ or $f l b A$ that results in formation of reduced conidiophores with spores forming directly from hyphal tips (Adams et al. 1988; Lee and Adams 1994b). Because flbD mRNA is present at relatively constant levels in vegetative hyphae and after development initiates in wild-type cells, we propose that the activity of the putative FlbD DNA-binding protein is regulated post-transcriptionally in response to early sporulation signals resulting in the transcriptional activation of other developmental regulators, such as brlA.

\section{Results \\ flbD is required for normal timing of conidiophore production}

Figure 1 shows the growth of wild-type (FGSC26) and flbD mutant (TJW30.1) colonies 2 and 3 days after inoculation on complete medium. Conidiophores were observed within the center of the wild-type colony by $24 \mathrm{hr}$ after inoculation, and by two days after inoculation conidiophores were seen throughout the colony (Fig. 1A). In contrast, no conidiophores were observed in the fluffy flbD mutant colony $24 \mathrm{hr}$ after inoculation and two days after inoculation only a small number of conidiophores were observed within the center of the colony (Fig. 1C). By 3 days postinoculation the area of central conidiation in the $f l b D$ mutant colony had expanded so that conidiophores were present throughout a larger area (Fig. 1D). The $f 1 b D$ mutant colony remained somewhat fluffy, however, and the margin of undifferentiated hyphae at the edge of the colony was $10-12 \mathrm{~mm}$ greater than the 1to $2-\mathrm{mm}$ margin of nonconidiating vegetative hyphae seen at the edge of the wild-type colony.

\section{Isolation and mapping of the flbD gene}

The wild-type $f l b D$ gene was isolated by complementation of an $f l b D^{-}$mutant as described in Yelton et al. (1984). RBN070 (biA1; argB2;flbD14) was transformed with an $A$. nidulans genomic DNA library made in a 


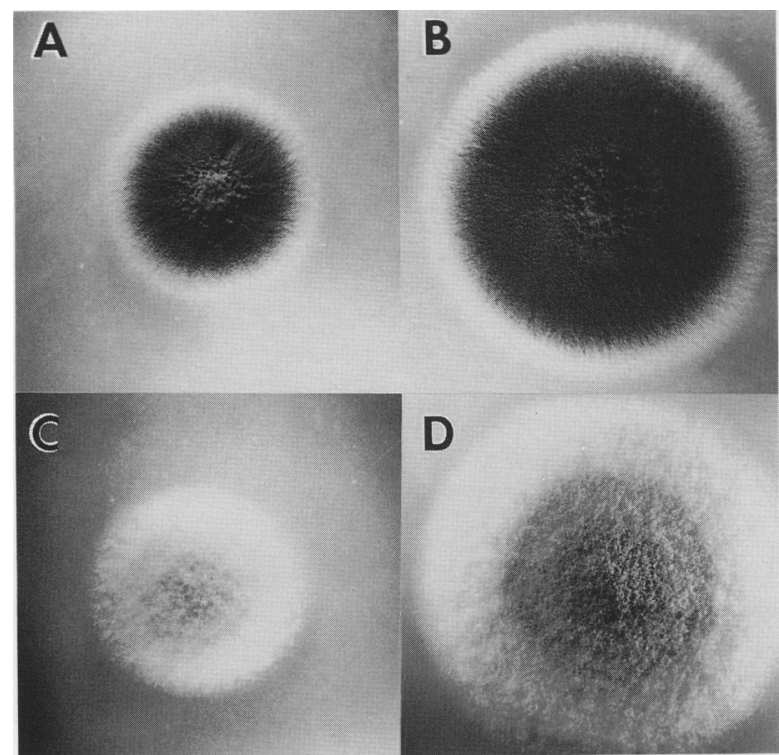

Figure 1. $f l b D$ is required for normal timing of conidiophore development. A wild-type strain (FGSC26;A,B) and an $f l b D$ mutant $(T J W 30.1 ; C, D$ ) were point inoculated on complete medium and allowed to grow for $48 \mathrm{hr}(A, C)$ or $72 \mathrm{hr}(B, D)$. The wild-type and mutant strains were photographed at the same magnification.

cosmid vector containing the wild-type $\arg B^{+}$gene to use as a selectable marker (pUI1; kindly provided by Dr. Bruce Miller, University of Idaho, Moscow). Approximately $2000 \mathrm{arg} B^{+}$transformants were selected on minimal medium lacking arginine, and two developmentally wild-type transformants were identified by visual screening (TJW001 and TJW002). Twenty-seven cosmids with identical restriction patterns were recovered following in vitro packaging of genomic DNA from TJW002 (Yelton et al. 1985), and three of these cosmids (designated $\mathrm{pJW} 8, \mathrm{pJW} 9$, and $\mathrm{pJW10}$ ) were selected for further analyses. Two of the recovered cosmids, pJW8 and pJW10, complemented the $f 1 b D 14$ mutation in RBN070 at high frequency indicating that they probably contain the entire wild-type $f l b D$ locus. Although pJW9 had identical restriction enzyme digestion patterns to pJW8 and $\mathrm{pJW10}$, it did not rescue the $\mathrm{flbD} 14$ mutation, suggesting it contained the $f l b D 14$ mutant allele. The $f l b D$ complementing region in pJW10 was localized to a 3.2$\mathrm{kb}$ BamHI-KpnI fragment as described in Materials and methods (Fig. 2).

\section{Disruption of the flbD gene results in a delayed conidiation fluffy phenotype}

To determine whether the cloned sequences corresponded to the wild-type $f l b D$ gene, we disrupted the putative $f l b D$-coding region by transformation of a developmentally wild-type strain (FGSC237) with a plasmid containing the entire $\operatorname{trp} C$ gene inserted into a unique $\mathrm{XhoI}$ site located within the $f l b D$ gene, $401 \mathrm{bp}$ downstream of the predicted FlbD translational start site (Fig. 2A,B; see results below). Seven of the $42 \operatorname{trp} C^{+}$ transformants had a delayed conidiation phenotype like that observed for the original flbD14 mutant strain (RBN021). Southern blot analysis with genomic DNA from these transformants showed that all seven delayed conidiation mutants tested contained the predicted insertion, whereas all $4 \operatorname{trp} C^{+} f l b D^{+}$transformants examined maintained the wild-type $f l b D$ gene. Despite repeated attempts, we have not been able to construct a heterozygous diploid strain between the $f l b D 14$ mutation and the putative $f l b D$ disruption mutant to determine allelism. However, the two mutations did not complement in heterokaryons, and when one $f l b D$ insertion mutant (TJW30.1) was crossed with RBN070 (flbD14), all 500 meiotic progeny analyzed had a delayed conidiation phenotype. This result indicates that the cloned region is closely linked to the original fIbD14 mutation, strongly supporting the idea that the $f l b D$ gene was disrupted in TJW30.1.

\section{FlbD is related to $c-m y b$}

The DNA sequence of the $f I b D$ gene region was determined and is presented in Figure 2B. The $f l b D$ gene is predicted to encode a 314-amino-acid polypeptide. We compared the predicted FlbD sequence with other sequences in various data bases using the BLAST search algorithm (Altschul et al. 1990) and found that the first 108 amino acids shared $>40 \%$ identity with the DNAbinding domains found in several transcription factors typified by the human proto-oncogene c-myb (Fig. 2C) (Majello et al. 1986). This region contains two or three imperfect repeats with 51 or 52 amino acids that typically include three critical tryptophan residues spaced either 18 or 19 residues apart (Lüscher and Eisenman 1990; Saikumar et al. 1990). The related region of FlbD contains two repeats, but each contains only two tryptophans with a tyrosine found at the position expected for the third tryptophan in the first repeat and a isoleucine found at the position expected for the first tryptophan in the second repeat (Fig. 2C). Both of these substitutions have been observed in other Myb-like proteins that remain functional as transcriptional activators (PazAres et al. 1987; Tice-Baldwin et al. 1989). Other residues in the Myb-like repeats that have been shown to be important in DNA binding were also conserved, including a highly conserved cysteine residue in the first repeat that has been shown to have a role in redox regulation of Myb DNA binding (Frampton et al. 1991; Guehmann et al. 1992; Myrset et al. 1993). Sequence analysis of the $f l b D$ coding region in pJW9, the recovered cosmid proposed to contain the flbD14 mutant allele (see above), indicated that the mutation resulted from a single basepair change converting glutamic acid 67 in the conserved DNA-binding domain to a lysine (Fig. $2 \mathrm{C}$ ). No significant similarities were observed between the remainder of the $f l b D$ coding region and other sequences in the various data bases. 
A

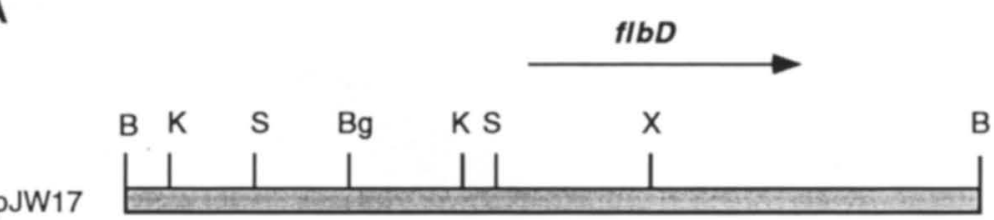

Figure 2. Restriction map and nucleotide sequence of the $f I b D$ gene and the deduced primary structure of the protein. $(A)$ The restriction map of pIW17 containing the $f l b D$ gene is shown. The direction of $f l b D$ transcription was deduced by analysis of a unidirectional cDNA clone and is indicated by the arrow positioned to represent the approximate $f l b D$ gene region. A $4.1-\mathrm{kb}$ Xhol $t r p C$ fragment was inserted into the $X$ hol site to disrupt the $f l b D$ gene. Restriction sites are abbreviated: $(B) B a m H I ;(K) K p n I ;(S)$ SalI; (Bg) BglII; (X) Xho. (B) The DNA sequence of the 2001-bp region corresponding to $f l b D$ and the predicted amino acid sequence of the FlbD protein are shown. The first $376 \mathrm{bp}$ and the last $207 \mathrm{bp}$ were derived from the genomic clone, and the rest from the cDNA clone. The lack of introns was confirmed by sequencing the genomic clone. The arrow indicates the site at which the cDNA sequence begins, and the asterisk (*) indicates the end of the cDNA clone. The XhoI site used for constructing the insertion mutant is underlined. The shaded region idicates the Myb-like domain shown in $C$. The GenBank accession number for this sequence is U19882. (C) The predicted amino-terminal amino acid sequence of FlbD is compared with that of various Myb-like proteins: Homo sapiens c-Myb (Majello et al. 1986; Slamon et al. 1986); Drosophila melanogaster $\mathrm{Myb}$ (Katzen et al. 1985; Peters et al. 1987); Arabidopsis thaliana GLl (Oppenheimer et al. 1991); Zea Mays C1(Paz-Ares et al. 1987); Schizosaccharomyces pombe CDC5 (Ohi et al. 1994); S. cerevisiae BAS1 (Tice-Baldwin et al. 1989). The two repeating motifs of $\sim 50$ amino acids are grouped, and the amino acids identical in five or more of the seven proteins shown are marked by the asterisks $\left({ }^{*}\right)$. Among these, conserved amino acids are underlined. The $\mathrm{K}$ indicates glutamic acid 67 , which was converted to a lysine in the $f 1 b D 14$ mutant allele. Conserved tryptophan residues are indicated by shaded boxes.

B

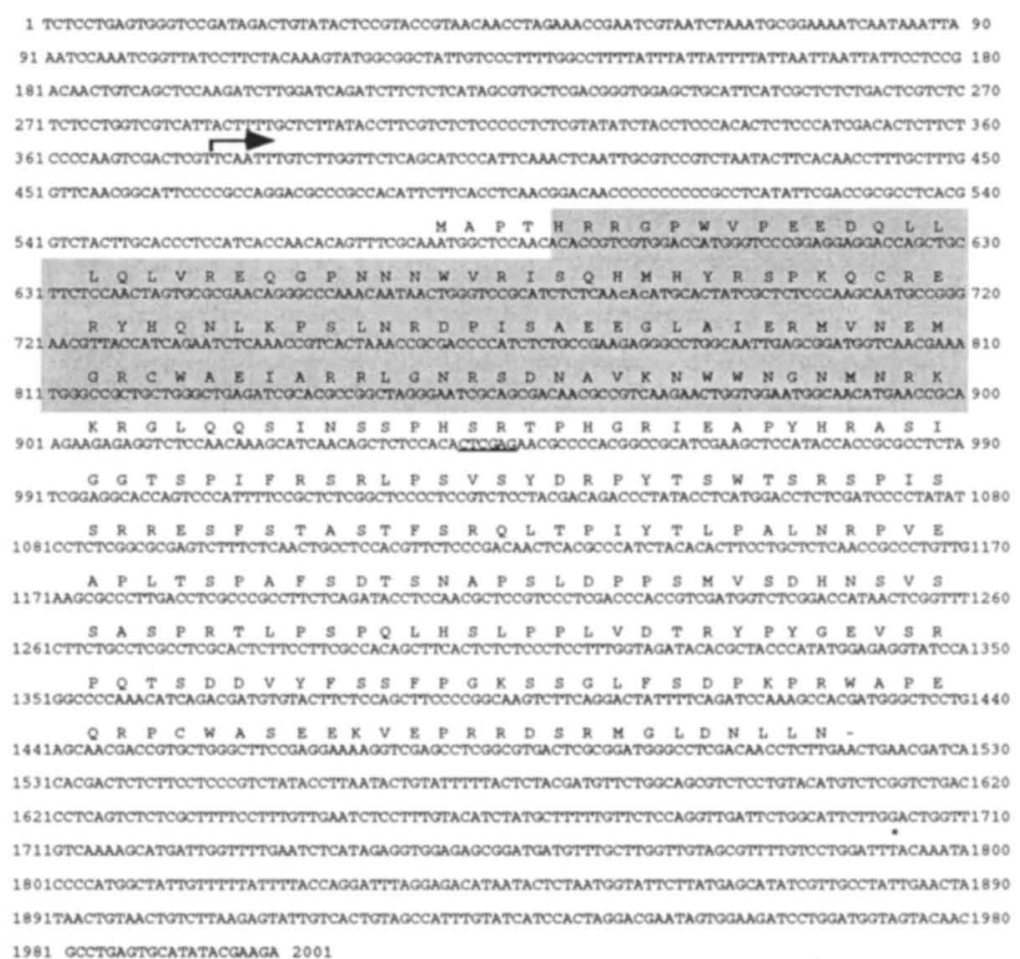

... *. * * *. *...... *.

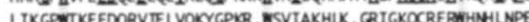
L TXK nax WO GLIIVE EDH L

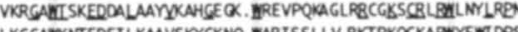
LKGGABKNTEDEI LKAAYSKYGXNQ DARISSLLV RKTPKOCKABRYYEIDES LKKGXEIQEEDEOLLKA YEEHGPHML SI SMOIP . . GRTELQCAKBYIEVLGE

REPEAT 2
A. nid. F1bD
H. sap. C-Myb
O. mel. myb
A. thal. GL1
2. mays C1
5. pombe Cde5
S. cer. BAS1

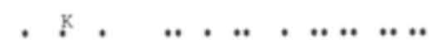
LNRDPISAEEGLAIERMWEMGRCEAEIARRLG NBSDAAVQNWGMAROK VKKTSETEEEDRI I YQAHKRLGPVAE IAKLLP GRTDNAIWN MNSTMFRX

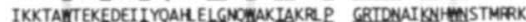
VNKGNTEKCIT

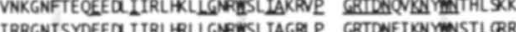

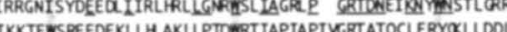
IKKTERS REEDEKLLHLAKLLPTOWRTIAPTAPIVGBIATQCLERYXLLDOL GRLREITLEEDLNLISWIKAYGTKWPKISSEIE FBPSLTREFARKIITM
$\mathrm{flbD} m R N A$ is present throughout the asexual life cycle of A nidulans

The $f l b D$ gene is apparently required specifically for conidiophore development and has no obvious role during hyphal growth (Wieser et al. 1994). We used a radiolabeled $f l b D$-specific fragment to probe total RNA isolated from submerged mycelial cultures and from cultures at various times after development was induced by exposing hyphae to an air interface, to investigate the possibility that $f l b D$ transcript accumulation is developmentally controlled. Figure $3 \mathrm{~A}$ shows that an $\sim 1.6 \cdot \mathrm{kb}$ transcript is present at relatively constant levels in both vegetatively growing and developing cells of wild-type $A$. nidulans. This transcript was absent in the $f l b D$ insertion mutant but a new $\sim 1-\mathrm{kb}$ transcript appeared. This is the size predicted if the transcription of full-length $f l b D$ message is blocked by the termination region in the $\operatorname{trp} C$ coding sequence inserted into flbD (Fig. 2A,B).

\section{brlA expression is delayed in flbD mutants}

The $f l b D$ mutant was isolated in a screen to identify fluffy mutants having reduced $b r l A$ expression as deter- 


\section{0}

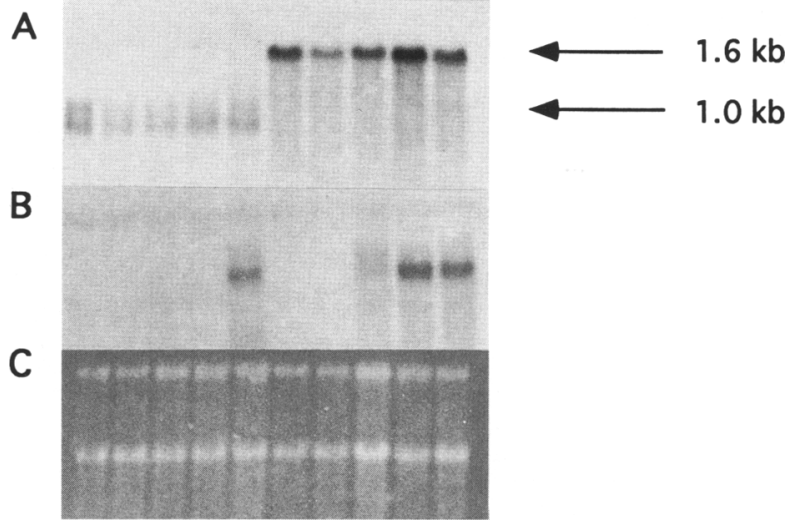

Figure 3. $f l b D$ mRNA is present throughout the $A$. nidulans life cycle and is required for normal timing of brlA expression. (A) Total RNA was isolated from vegetative and developing cultures of an $f l b D$ insertion mutant (T)W30.1, lanes 1-5) and from a wild-type strain (FGSC26, lanes 6-10) and fractionated on a formaldehyde-agarose gel. The resultant gel blot was probed with an $f l b D$-specific fragment $(A)$ or with a brlA-specific fragment $(B)$. RNA was isolated $22 \mathrm{hr}$ after inoculation (lanes 1,6$), 4$ $\mathrm{hr}$ after developmental induction (lanes 2,7), $8 \mathrm{hr}$ after developmental induction (lanes 3,8), $12 \mathrm{hr}$ after developmental induction (lanes 4,9), and $24 \mathrm{hr}$ after developmental induction (lanes $5,10)$. Relative sizes of mRNA are indicated at right. $(C)$ Equal loading of total RNA was confirmed by ethidium bromide staining.

mined by measuring brlA promoter-directed lac $Z$ expression. Less than $5 \%$ of wild-type brlA-directed $\beta$-galactosidase activity was detected in $f l b D$ mutant strains following developmental induction (Wieser et al. 1994). To confirm that $f l b D$ influences the timing of brlA transcript accumulation, we isolated total RNA from wildtype and $f l b D$ mutant strains at various times after induction and examined brlA expression patterns (Fig. 3B). brlA message first appeared in the wild-type strain between 4 and $8 \mathrm{hr}$ after developmental induction and accumulated to higher levels as development proceeded. In contrast, no brlA mRNA was detected in the $f l b D \mathrm{mu}$ tant strain until $24 \mathrm{hr}$ after developmental induction. This corresponded to the time when conidiophores were first observed in the $f l b D$ mutant.

\section{Overexpression of $\mathrm{flbD}$ causes production of a complex} conidiophore in submerged culture

Conidiophore development is normally suppressed when $A$. nidulans is grown in submerged culture (Axelrod 1972; Law and Timberlake 1980). However, we have shown that other genes that have been identified in $A$. nidulans based on their requirement during normal conidiophore production are able to cause developmental induction inappropriately when overexpressed in submerged hyphae (Adams et al. 1988; Lee and Adams 1994b). To determine if $f l b D$ could activate conidiation in submerged culture, we constructed strains containing one (TJW29.2) to several (TJW29.4) copies of the flbD gene fused to the nutritionally regulated alc $A$ promoter $|a l c A / p /|$ and integrated into the $A$. nidulans genome at the $\operatorname{trp} C$ locus, leaving the wild-type $f l b D$ gene intact. alc $A$ transcription is repressed in the presence of glucose and induced in the presence of threonine (Lockington et al. 1985; Gwynne et al. 1987). As expected, fIbD mRNA could be detected in both TJW29.2 and TJW29.4 grown in glucose medium, but the $f I b D$ message levels in creased at least fourfold by $3 \mathrm{hr}$ after transferring TJW29.2 and TJW29.4 to threonine medium and remained high at all time points examined (not shown). $f l b D$ message could also be detected in both TTA11 (wild-type) and TTA292 (alcA(p)::brlA) grown in glucose medium, but the level of $f l b D$ expression did not increase in either strain after the shift to threonine medium, and $f l b D$ mRNA levels may have even decreased slightly in TTA292 (not shown).

brlA mRNA normally accumulates to detectable levels only after induction of development and has been shown to be required under all conditions for the production of conidiophores (Clutterbuck 1969; Adams et al. 1988). We therefore determined the effect that overexpression of $f l b D$ had on brlA expression. Figure 4 shows that in TJW29.2, brlA message could be detected by $6 \mathrm{hr}$ after a shift to alc $A$-inducing medium, whereas in TJW29.4, brlA message could be detected $3 \mathrm{hr}$ after the shift to induction medium. brlA message was also detected in TTA292 by $3 \mathrm{hr}$ after the shift to alcA-inducing medium but was never detected in the wild-type (TTAl1) strain grown in submerged culture.

Overexpression of $f l b D$ in hyphae growing in submerged culture resulted in rapid cellular changes that culminated in the production of complex conidiophores that included stalks, vesicles, metulae, phialides, and co-

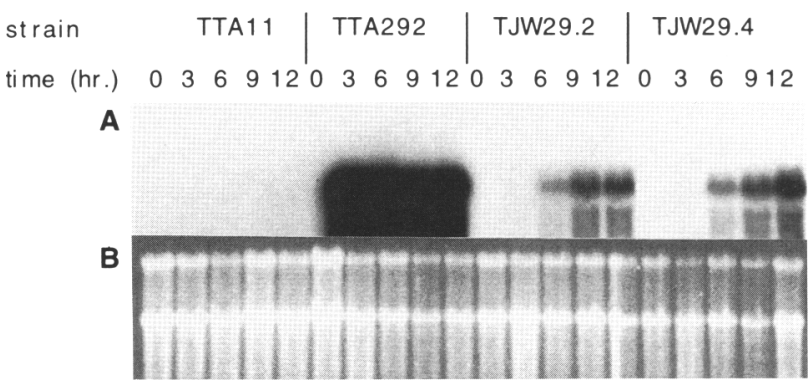

Figure 4. Forced overexpression of $f l b D$ results in accumula tion of brlA mRNA. (A) Total RNA was isolated from a developmentally wild-type $A$. nidulans strain (TTAl1) or from strains containing an alcA(p)::brlA fusion (TTA292), multiple copies of the alcA(p)::flbD fusion (TJW29.4), or a single copy of the $\operatorname{alcA}(p):: f l b D$ fusion (TJW29.2). The strains were grown in liquid minimal medium containing glucose for $14 \mathrm{hr}$ (labeled 0 $\mathrm{hr}$ ) or shifted from this medium to minimal medium with threonine and allowed to grow for an additional $3 \mathrm{hr}, 6 \mathrm{hr}, 9 \mathrm{hr}$, or $12 \mathrm{hr}$. The resulting gel blot was probed with a brlA-specific fragment. $(B)$ Equal loading of total RNA was confirmed with ethidium bromide staining. 
nidia. Figure 5B shows that by $3 \mathrm{hr}$ after alcA-induced expression of $f l b D$, the hyphae had become highly vacuolated. By $9 \mathrm{hr}$ after induced overexpression of $f l b D$, simple conidiophores were formed consisting of swellings at the tips of hyphae from which spores were produced (Fig. 5D), and by $18 \mathrm{hr}$ after shifting to alcA-inducing medium, mophologically complex spore-producing conidiophores were present throughout the culture (Fig. 5E). These conidiophores contained all of the cell types seen in normal wild-type conidiophores including stalks, vesicles, sterigmata, and spores (Fig. 5, cf. E and J). The phenotypes of TJW29.2 and TJW29.4 were the same at all time points (not shown). By $3 \mathrm{hr}$ after a shift to threonine medium, TTA292 (alcA(p)::brlA) became vacuolated and produced reduced conidiophores in which a spore formed directly from the hyphal tip (Fig. 5G). The TTA292 culture did not change with time and looked much the same at $18 \mathrm{hr}$ after the shift to inducing medium as it did $3 \mathrm{hr}$ after the shift from glucose to threonine medium (Fig. 5H). In contrast to TJW29.2, TJW29.4, and TTA292, the wild-type strain, TTA11, did not pro-

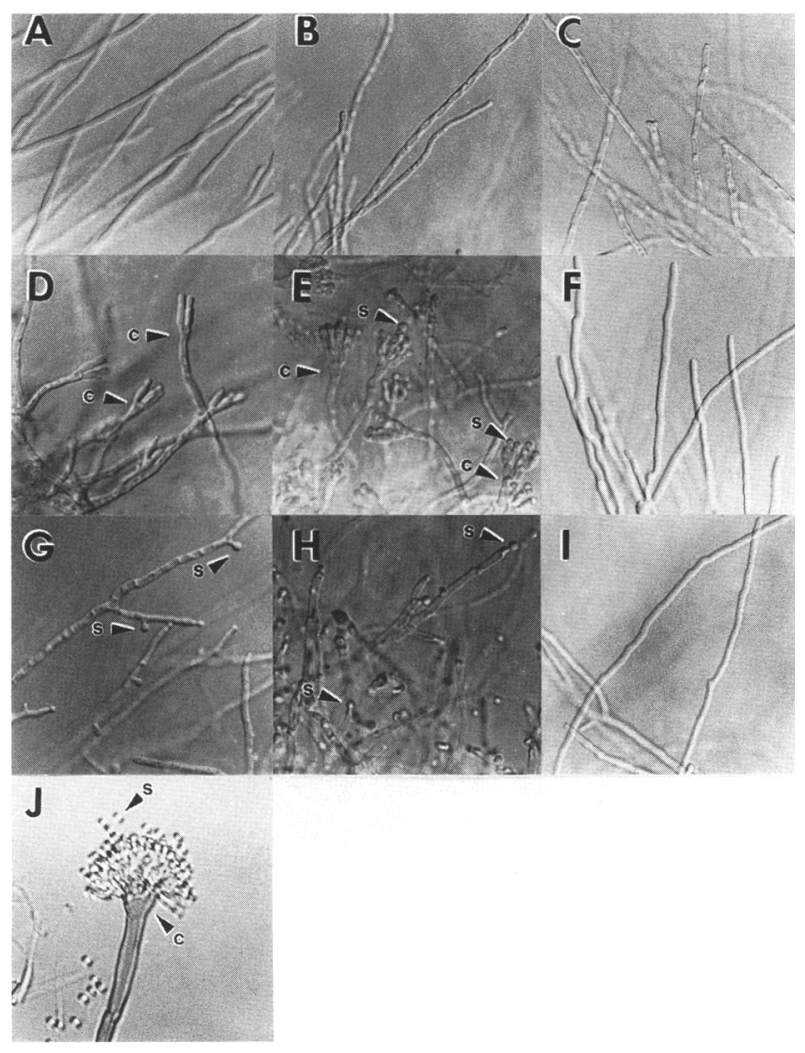

Figure 5. Overexpression of $f l b D$ causes conidiophore development. Strains TJW29.2 [alcA(p):: flbD (A-E)], TTA292 |al$c A(p):: b r l A(F-H)]$, and TTAII [wild-type (I)] were grown in liquid minimal medium with glucose $(A, F)$ and then shifted to minimal medium containing threonine and allowed to grow for an additional $3 \mathrm{hr}(B, G), 6 \mathrm{hr}(C), 9 \mathrm{hr}(D)$, or $18 \mathrm{hr}(E, H, I)$. (J) A wild-type conidiophore produced by TAll grown on the surface of an agar plate. Specimens were photographed using DIC optics. Arrows indicate conidiophores (c) or spores (s). duce any conidiophores and the hyphae did not undergo any morphological changes other than those that occur during normal growth (Fig. 5I).

\section{Genetic requirements for flbD-induced conidiation}

By constructing double mutants in all pair-wise combinations among the FLB mutants, we showed that $f l b B$, $f l b D$, and $f l b E$ probably function in the same step of a pathway leading to conidiation and that this pathway is separate from the pathway in which $f l b C$ functions (Wieser et al. 1994). In addition, we showed that $f l b A$ and $f l u G$ were epistatic to $f l b D$. The observation that overexpression of $f l b D$ in liquid culture resulted in developmental activation provided the opportunity to extend this classical epistatic data to include information on the genetic requirements for $f l b D$-overexpression-induced development. We therefore constructed developmental mutant strains that contained the alcA(p)::flbD fusion and assessed the effects of $f l b D$ overexpression in these strains. Forced overexpression of $f l b D$ in liquid-grown hyphae failed to cause conidiophore production in $b r l A^{-}$ (RJW223), flbA $A^{-}$(RJW193), and $f l b B^{-}$(RJW201) strains that contained a single copy of the alcA(p)::flbD fusion construct (Fig. 6B,C,G; Table 1). For all of these strains except the $f l b B^{-}$strain (RJW201), there were extreme changes in hyphal morphology including increased vacuolation and severe growth inhibition by $3 \mathrm{hr}$ after shifting to alcA-inducing medium. Overexpression of $f l b D$ in an $\mathrm{flbE}^{-}$mutant (RJW197) led to the production of conidiophores with the same timing as observed in TJW29.2; however, conidiophore structures tended to be somewhat simpler (Fig. 6E). Overexpression of $f 1 b D$ in the $\mathrm{flbC}^{-}$mutant (RJW207; Fig. 6D) always resulted in very vacuolated hyphae, but the conidiophores produced were not as numerous nor as complex as those produced in the wild-type strain containing the alc $A(p):: f l b D$ construct (TJW29.2; Figs. 5 and 6H). Likewise, forced overexpression of $f l b D$ in a flu $G^{-}$mutant (RJW194; Fig. 6F) resulted in conidiophore production that was neither as extensive nor as rapid as that observed in the wild-type background. As summarized in Table 1, $f l b D$-induced conidiophore development was always associated with accumulation of brlA mRNA.

\section{Discussion}

$A$. nidulans strains bearing mutations in any of four different genes, $f l b B, f l b C, f l b D$, and $f l b E$, have a temporally altered developmental program resulting in fluffy colonies that, like wild-type strains, produce conidiophores within the center of the colony (Wieser et al. 1994). However, conidiation is delayed at least $24 \mathrm{hr}$ in these mutants as compared with developmentally wildtype strains (Fig. 1). Genetic analysis of these delayed conidiation mutants indicated that they probably define separate branches of the pathway leading to developmental activation, with $f l b B, f l b D$, and $f l b E$ defining one branch and flbC defining the second (Wieser et al. 1994). Whereas elimination of one branch of the pathway 


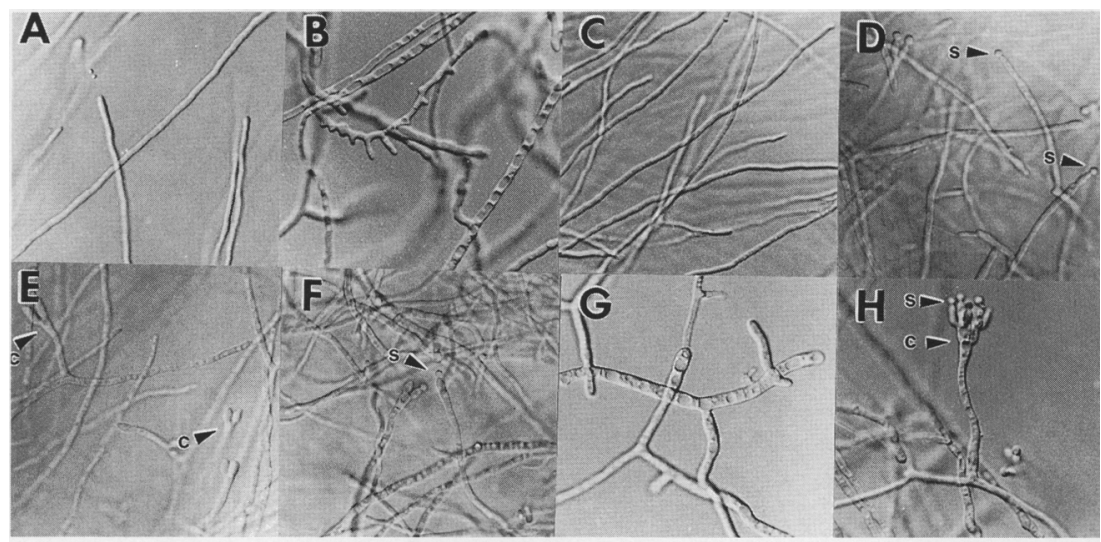

Figure 6. Overexpression of $f l b D$ in developmental mutant strains. Strains TTA1l [control $(A)]$, RJW193 [ $\triangle f l b A:$ alcA $(p):: f l b D$ $(B)]$, RJW201 $\left[f l b B^{-}\right.$, alcA(p)::flbD $\left.(C)\right]$, RJW207 [flbC $\left.{ }^{-}, a l c A(p):: f l b D(D)\right]$, RJW197 $\left[f l b E^{-}, a l c A(p):: f l b D(E)\right]$, RJW194 [DfluG, alcA $(p):: f l b D(F)]$, RJW223 [ $\Delta b r l A$, alcA $(p)$ $\therefore f l b D(G)]$, and TJW29.2 [alcA(p)::flbD $(H)]$ were grown in liquid minimal medium containing glucose for $14 \mathrm{hr}$ and then shifted from this medium to liquid minimal medium with threonine and allowed to grow for $12 \mathrm{hr}(A, B, D, E, G, H)$ or $24 \mathrm{hr}(D, F)$. Specimens were photographed using DIC optics.

causes a delay in conidiation, elimination of both branches causes a longer delay. However, conidiophores are still produced by the double mutant indicating that development can also be activated through other mechanisms (Adams et al. 1992; Wieser et al. 1994). We have shown here that at least one of these delayed conidiation genes, $f l b D$, is directly involved in activating conidiophore development, because forced overexpression of $f l b D$ in submerged culture, a condition that normally inhibits conidiation, resulted in the formation of complex conidiophores that produced viable spores. These conidiophores had all of the cell types observed in normal conidiophores including stalks, vesicles, metulae, phialides, and spores, leading us to propose that $f l b D$ normally functions early in development, resulting in activation of all of the genes necessary to make a complete conidiophore.

The similarity observed between the amino-terminal portion of the predicted FlbD protein and the DNA-binding domain for proteins in the Myb family of transcription factors suggests that the function of $f l b D$ in development involves directly regulating transcription of other genes (Lüscher and Eisenman 1990). Like Myb,

Table 1. Effects of developmental mutations on the flbD overexpression phenotype

\begin{tabular}{|c|c|c|c|}
\hline Strain $^{a}$ & $\begin{array}{l}\text { brlA } \\
\text { expression }\end{array}$ & Sporulation & Vacuolation \\
\hline RJW193 (flbA-) & - & - & + \\
\hline RJW201 $\left\{f l b B^{-}\right\}$ & - & - & - \\
\hline RJW207 $\left(f 1 b c^{-}\right)$ & + & + & + \\
\hline RJW197 $\left|f 1 b E^{-}\right|$ & + & + & + \\
\hline RJW194 (fluG ${ }^{-}$) & + & + & + \\
\hline RJW233 (brlA $\left.{ }^{-}\right)$ & - & - & + \\
\hline TJW29.2 (wild type) & + & + & + \\
\hline TTA11 (control) & - & - & - \\
\hline
\end{tabular}

Strains were grown as described in Materials and methods and were observed at various times beginning $3 \mathrm{hr}$ after induction and continuing through $24 \mathrm{hr}$ postinduction.

${ }^{a}$ All strains contain a single copy of the $\operatorname{alc} A(p):: f l b D$ fusion except the control strain TTAll.
FlbD apparently regulates the transition from cell proliferation to differentiation of new cell types. Because loss of $f l b D$ function results in a delay in conidiophore development and extensive uncontrolled growth, whereas overexpression of $f I b D$ in hyphae causes conidiophore development at inappropriate times, it seems likely that FlbD has a positive role in activating development-specific gene expression. Given that the early developmental regulatory gene $b r l A$ is required for $f l b D$ to cause production of conidiophores in submerged culture, one possible downstream target of FlbD is brlA. The brlA locus is complex, consisting of two overlapping transcripts designated $b r l A \alpha$ and $b r l A \beta$, both of which are required for normal conidiophore development (Han et al. 1993; Prade and Timberlake 1993). We showed that the delay in conidiation observed in $f l b D$ mutants was accompanied by a similar delay in accumulation of $b r l A$ mRNA, whereas forced overexpression of $f l b D$ resulted in the inappropriate activation of $\operatorname{brl} A \alpha$ and $b r l A \beta$ expression (Fig. 4; data not shown). However, we have not yet demonstrated direct control of $b r l A \alpha$ or $b r l A \beta$ transcription by $f l b D$.

Because overexpression of $f l b D$ in $b r l A$ mutants is able to cause cellular changes that resemble those occurring following induction of normal development in $b r l A$ null mutants, we consider it likely that if brlA is regulated directly by FlbD, it is likely not the only such gene. The observation that overexpression of $f l b D$ in a wild-type strain resulted in formation of morphologically normal conidiophores whereas overexpression of brlA resulted in development of much simpler conidiophore-like structures also supports this hypothesis. The higher complexity of the conidiation structures produced following overexpression of $f l b D$ presumably reflects differences in timing, extent, and spatial organization of gene expression, but how these differences affect development will require identification of the specific targets for FlbD function.

If FlbD has a development-specific function in activating transcription of other genes like brlA, it is important to know how the activity of FlbD is controlled. As with other genes identified by fluffy mutations, $f l b D$ mRNA 
accumulates to relatively constant levels throughout the A. nidulans life cycle (Adams et al. 1992; Lee and Adams $1994 \mathrm{a}, \mathrm{b})$. This might indicate that $f l b D$, like $c-m y b$, is probably regulated post-transcriptionally (Lüscher and Eisenman 1990). This brings up the question of why forced overexpression of $f I b D$ causes development in submerged culture. One explanation for this result could be that FlbD alone has a basal activity but it requires the products of other genes to reach the level required for developmental induction. If this were the case, overexpression of $f l b D$ mRNA could bypass the need for these other activities by increasing the concentration of $\mathrm{FlbD}$. Similarly, the delay observed for conidiophore development in $f l b D$ loss-of-function mutants might be explained if the products of other genes can replace the need for FlbD once they have attained sufficient levels. Along these lines, it is interesting that the one other delayed conidiation mutant we have begun to characterize also results from loss of function in a gene $(f l b C)$ that encodes a putative transcription factor (J. Fondon and T.H. Adams, unpubl.). FlbC and FlbD apparently contribute independently to developmental activation because mutations in both $f l b C$ and $f l b D$ have an additive effect on development and $f l b C$ is not required for overexpression of $f l b D$ to cause development (Table 1). A second possible mechanism for regulating FlbD activity is through changes in the environment. Under normal conditions, $A$. nidulans does not go through conidiophore development unless hyphae are exposed to an air interface (Morton 1961; Axelrod et al. 1973; Timberlake 1980). This is particularly relevant because it has been demonstrated that changes in redox state can induce conformation changes in the Myb DNA-binding domain, altering its ability to stably associate with DNA /Guehmann et al. 1992; Myrset et al. 1993). Given that the change in redox is apparently sensed through a conserved cysteine residue in the first repeat for the DNA-binding domain shown in Figure 2C, one possible explanation for the role of air in A. nidulans development is in controlling the activity of FlbD. In this model, increased expression of FlbD in submerged culture must in some way overcome the need for redox-induced changes in DNA binding.

To address the question of how FlbD activity is regulated, we have investigated the genetic requirements for FlbD-induced development, and a model summarizing the potential interactions between FlbD and other genes controlling brlA activation is presented in Figure 7. We proposed previously that FluG is responsible for the constitutive production of an extracellular signal for conidiation and that development only occurs once a threshold level of this FluG-dependent signal has been reached (Lee and Adams 1994a). This hypothesis is consistent with the fact that forced overexpression of $f l b D$ does not absolutely require flu $G$ for the production of conidiophores in liquid (Fig. 6; Table 1). However, the conidiophores produced in a flu $G^{-}$mutant following $f l b D$ overexpression are not as complex or as numerous as those produced following $f I b D$ overexpression in a wild-type strain. This might be explained if the FluG activity contributes to activation of FlbD but is not absolutely required for it. For instance, FluG signal may be involved in modulating the activity of other genes (such as $f l b A$ ) that work in conjunction with FlbD to activate development.

Analysis of double mutant strains lacking combinations of $f l b D, f l b B, f l b C$, and $f l b E$ led to the hypothesis that $f l b B, f l b D$, and $f l b E$ function at the same step in a genetic pathway leading to the activation of $b r l A$ and that $f 1 b C$ functions in a separate pathway (Wieser et al. 1994). Because $f l b E$ is not required for overexpression of $f l b D$ to cause development in submerged culture but $f l b B$ is necessary, we can refine this model to include the gene order $f l b E \rightarrow f l b D \rightarrow f l b B$ for one branch of the

Figure 7. Genetic model for the role of $f I b D$ in regulating development. Our previous results led us to suggest that the flu $G$ gene product is responsible for producing a signal that functions extracellularly in activating a pathway leading to conidiation and that the $f 1 b A$ product regulates development directly by controlling the response to this signal (Lee and Adams 1994a,b). The data presented in this paper indicate that $f l b D$ also has a direct role in activating $b r l A$ and development because overexpression of $f l b D$ is sufficient to cause sporulation in submerged culture. On the basis of epistasis analysis, $f l b E$ and $f l b B$ probably

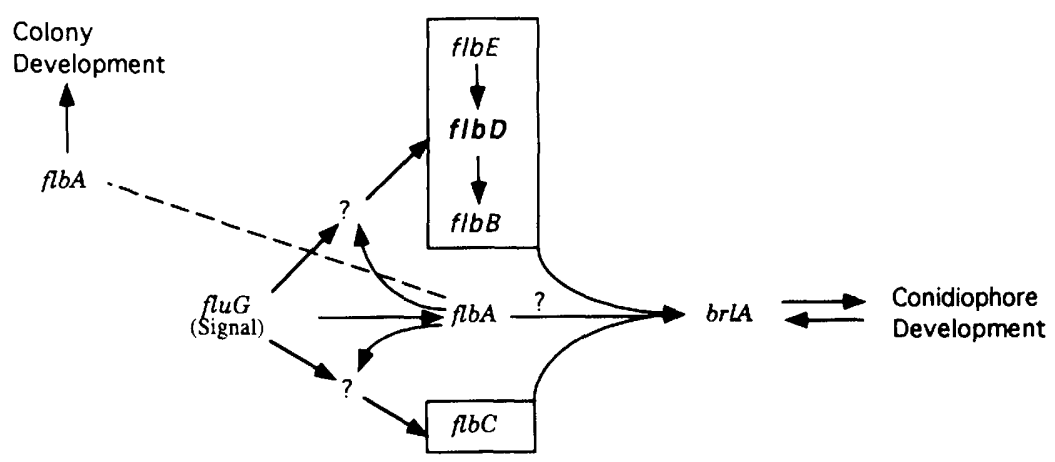
function at the same step as $f l b D$ in the genetic pathway leading to the activation of $b r l A$ and development, whereas $f l b C$ may function in a separate branch of the same pathway (Wieser et al. 1994). Because $f l b B$ is required for $f I b D$-induced sporulation but $f l b E$ is not, we suggest that $f l b E$ acts before $f l b D$ whereas $f l b B$ functions downstream of $f l b D$. fluG mutations are epistatic to $f l b D$ mutations (Wieser et al. 1994), and flu $G$ is not needed for $f l b D$ overexpression to cause development as expected if flu $G$ functions before $f l b D$ in regulating development. The placement of $f l b A$ in this pathway is somewhat uncertain. $f l b A$ mutations are epistatic to $f l b D$ mutations, but $f l b A$ is required for overexpression of $f l b D$ to activate development. We propose that $f l b A$ functions upstream of $f l b D$ in positively modulating FlbD activity, although it is also possible that $f l b A$ functions later in the pathway. Finally, because $f l b A$ mutations also affect vegetative growth, we also propose a second role for $f l b A$ in controlling aspects of colony development unrelated to conidiophore development. 
pathway (Table 1; Fig. 7). The nature of these interactions will be made more clear by examining the roles of $f l b B$ and $f l b E$ in development more directly. As mentioned above, forced overexpression of $f l b D$ does not require $f l b C$ to cause conidiation in submerged culture although the number and complexity of the conidiophores produced is reduced in the $f l b C$ mutant. This result is consistent with $f l b C$ functioning in a separate pathway from $f l b D$ leading to the activation of $b r l A$. Loss of the $f l b C$ pathway can be partially compensated for by the $f l b E, f l b D, f l b B$ pathway, resulting in delayed conidiation. Likewise, the $f 1 b C$ pathway can partially compensate for the loss of $f l b D, f l b B$, or $f l b E$ function again resulting in a delayed conidiation phenotype. Because some development still takes place in the absence of both pathways, there must also be other mechanisms for activation of the central conidiation pathway.

Because FlbA is related to the $S$. cerevisiae SST2 gene product and SST2 functions in regulating the activity of the G-protein-mediated signal transduction pathway that controls yeast mating, we have suggested that FlbA could function by regulating the activity of a signal transduction pathway for responding to the putative fluG signal for conidiation (Lee and Adams 1994b). However, the relationship between $f l b A$ and the other genes that are normally required for activating $b r l A$ expression is somewhat complicated (Wieser et al. 1994). flbA mutations are epistatic to mutations in all of the other $f l b$ loci as predicted if $f l b A$ functions early in development, before $f l b B, f l b C, f l b D$, or $f l b E$. In contrast, $f l b A$ is required for conidiophore development induced by overexpression of $f I b D$ in submerged culture. This could be explained if $f l b A$ functions upstream of $f l b D$ and is required for bringing about events leading to FlbD activation. Alternatively, FlbD activity may be required for activation of $f l b A$. It is interesting that overexpression of $f l b A$, like overexpression of $f l b D$, has been shown to activate $b r l A$ expression and development in submerged culture. The structures produced following forced overexpression of $f l b A$ in submerged culture are much simpler than the complex conidiophores made following overexpression of $f I b D$ (Fig. 5), consisting of simple phialide-like structures bearing chains of spores (Lee and Adams 1994b). One interpretation of this difference in conidiophore complexity is that $f l b D$ functions upstream of $f l b A$ in regulating initiation of conidiophore development. However, $f l b D$ overexpression can result in brlA mRNA accumulation by $3 \mathrm{hr}$ after induction, whereas $f l b A$ overexpression does not cause brlA accumulation until between 6 and $9 \mathrm{hr}$ postinduction. Clearly, the details of these interactions require a more complete understanding of the functions of FlbD, FlbA, and the products of other genes regulating initiation of A. nidulans conidiophore development.

\section{Materials and methods}

Fungal strains, growth, and genetics

All A. nidulans strains used in this study are listed in Table 2.
MBNO2l is the original $f l b D^{-}$mutant strain and was isolated from FGSC26 following NQO mutagenesis (Wieser et al. 1994). RBN021 was isolated as the meiotic progeny of FGSC237 and MBN014 and was crossed to PW1 to yield RBN070, an $\operatorname{argB2^{-}}$, flbD14- mutant strain. TJW001 (argB $\left.B^{+} f l b D^{+}\right)$was isolated following transformation of RBN070 with an $A$. nidulans genomic DNA library constructed in the $\arg B^{+}$-containing cosmid vector pUIl (kindly provided by Dr. Bruce Miller, University of Idahol. TJW30.1 was constructed by transformation of FGSC237 with the $f l b D$ insertion plasmid pJW30. TJW29.2 and TJW29.4 were constructed by transformation of FGSC237 with the al$c A(p):: f l b D$ fusion plasmid pJW29. TJW29.2 has a single copy of pIW29 integrated at the $\operatorname{trp} C$ locus, whereas TJW29.4 has multiple copies of the plasmid integrated at $\operatorname{trpC}$. RJW193, RJW194, RJW197, RJW201, RJW207, and RJW223 were constructed by crossing TJW29.2 to TBN39.5, RBN119, RJW120, RJW012, RJF003, or RSH94.4, respectively, to produce $f l b A^{-}$, flu $G^{-}$, $f l b E^{-}, f l b B^{-}, f l b C^{-}$, and $b r l A^{-}$mutant strains, each containing a single copy of the $a l c A(p):: f l b D$ fusion construct integrated at the $\operatorname{trp} C$ locus as determined by Southern blot analysis.

Minimal medium with appropriate supplements was prepared as described (Käfer 1977). Complete medium is standard minimal medium with 5 grams of yeast extract per liter. Developmental cultures were grown on appropriately supplemented complete medium, and induction was performed as described previously (Han et al. 1993). For the $\operatorname{alc} A(p)$ induction experiments, spores were inoculated at a density of $1 \times 10^{6}$ spores $/ \mathrm{ml}$ in minimal medium containing $50 \mathrm{mM}$ glucose and shaken at $300 \mathrm{rpm}$ and $37^{\circ} \mathrm{C}$ for $14 \mathrm{hr}$. Aconidial mutant strains were inoculated using either a hyphal macerate or as $1 \times 10^{6}$ ascospores $/ \mathrm{ml}$ of culture. Following growth in liquid medium, hyphal cells were harvested by pouring through sterile Miracloth (Calbiochem), washed twice with minimal medium without glucose, transferred to minimal medium containing $100 \mathrm{~mm}$ L-threonine, and incubated as above. Samples were taken at the time points indicated for microscopic observation and RNA isolation.

Genetic techniques and microscopy

Standard genetic (Pontecorvo et al. 1953; Clutterbuck 1974) and transformation techniques (Yelton et al. 1984; Miller et al. 1985) were used. Photomicrographs of hyphal development were taken using an Olympus $\mathrm{BH} 2$ compound microscope and differential interference contrast optics.

\section{flbD sequence analysis}

Because $f l b D$ mRNA was present at constant levels in vegetative hyphae and following induction (Fig. 3), we were able to identify an $f l b D$ cDNA clone by screening an $A$. nidulans $\lambda$ UNIZAP cDNA library made from RNA isolated from a vegetatively growing wild-type culture (O'Donnel et al. 1991). The 3.2-kb fragment in pJW18 was used to screen $3 \times 10^{5}$ plaques, and three positive clones were identified and rescued as phagemids. pIW25 was determined to have a 1.6 -kb insert as observed for the $f l b D$ message, and the entire sequence was determined by the chain-termination method (Fig. 2B). The 3.2-kb fragment from pJWl 8 was also sequenced and found to be colinear with that of the cDNA. Finally, pJW9 was used to determine the sequence of the region corresponding to the FlbD14 mutant coding region.

\section{Nucleic acid isolation and manipulation}

Total RNA was isolated as described in Adams et al. (1988) and then was separated ( $10 \mu \mathrm{g}$ per lane) by electrophoresis on a form- 
Table 2. A. nidulans strains used in this study

\begin{tabular}{|c|c|c|}
\hline Strain & Genotype & Source \\
\hline TJW001 & biA1; veA1 & this study \\
\hline TJW002 & biA1; veA1 & this study \\
\hline TJW30.1 & pabaA1, yA2; flbD::trp $C^{+}, v e A 1$ & this study \\
\hline FGSC26 & biA1, veA1 & FGSC $^{a}$ \\
\hline TJW29.2 & pabaA1, yA2; trpC::alcA $(p):: f 1 b D$, veA1 & this study \\
\hline TJW29.4 & pabaA1, yA2; trpC::alcA(p)::flbD, veA1 & this study \\
\hline TTA11 & pabaA1, yA2; veA1 & Adams et al. (1988) \\
\hline TTA292 & $b i A 1 ; \arg B^{+}:: a l c A(p):: b r l A \alpha ;$ methG1; veA1 & Adams et al. $(1988)$ \\
\hline RJW193 & pabaA1, $\triangle f l b A:: a r g B ; \operatorname{trp} C:: a l c A(p): \because f l b D, v e A 1$ & this study \\
\hline RJW194 & pabaA1; $\Delta f l u G ; \operatorname{trp} C:: a l c A(p):: f l b D, v e A 1$ & this study \\
\hline RJW197 & pabaA1, yA1, flbE58; methG1; veA1 & this study \\
\hline RJW201 & pabaA1; flbB $; ;$ trpC::alcA $(p):: f 1 b D$, veA1 & this study \\
\hline RJW207 & $p a b a A 1 ; f l b C 8 ; \operatorname{trp} C:: a l c A(p):: f l b D, v e A 1$ & this study \\
\hline RJW223 & pabaA1; $\triangle b r l A, \operatorname{trp} C:: a l c A(p):: f l b D, v e A 1$ & this study \\
\hline TBN39.5 & biA1, $\triangle f 1 b A:: a r g B ;$ meth $G 1 ;$ veA1 & Lee and Adams (1994) \\
\hline RJW012 & $b i A 1 ; f l b B 7 ; v e A 1$ & Wieser et al. (1994) \\
\hline RJF003 & pabaA1, yA2; argB2; veA1; flbC8 & Wieser et al. (1994) \\
\hline MBN014 & biA1; flbD14, veA1 & Wieser et al. (1994) \\
\hline RBN021 & pabaA1, yA2; flbD14, veA1 & Wieser et al. (1994) \\
\hline RBN070 & biA1; $\arg B 2 ;$ flbD14, veA1 & Wieser et al. (1994) \\
\hline RBN119 & biA1, yA2; $\triangle$ fluG, argB2; methG1; veA1 & Lee et al. (1994) \\
\hline RSH94.4 & methG1; $\triangle b r l A, v e A 1$ & S.T. Han (unpubl.) \\
\hline RJW120 & flbE58; methG1; veA1 & Wieser et al. (1994) \\
\hline PW1 & biA1; $\arg B 2 ; \operatorname{met} G 1 ; \operatorname{veA1}$ & P. Weglenski (unpubl.) \\
\hline FGSC237 & pabaA1, yA2; $\operatorname{trp} C 801, v e A 1$ & $\mathrm{FGSC}^{\mathrm{a}}$ \\
\hline
\end{tabular}

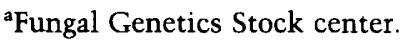

aldehyde-agarose gel and transferred to a nylon membrane ( $\mathrm{Hy}$ bond-N, Amersham Corp., Arlington Heights, IL). Hybridization to ${ }^{32} \mathrm{P}$-labeled random primed probes was done according to the procedures recommended by the manufacturer. A $1.9-\mathrm{kb}$ HindIII-SalI fragment from pTA39 containing the brlA coding sequence was used as a brlA-specific probe. A $1.3-\mathrm{kb} N c o$ I fragment from the cDNA clone pJW25 that contains the $f 1 b D$ coding sequence was used as the $f l b D$-specific probe. Hybridization to RNA blots was quantified using a Fujix BAS2000 phosphorimaging system.

The $f l b D$ gene region was localized in the following way: Eight $B a m H I$ subclones were made from pJW10, the cosmid that originally complemented the $f l b D$ mutation, by digesting p/W10 with $B a m H I$ and inserting each of the eight fragments into pPK1, a vector that contains the $\arg B$ gene as a selectable marker for Aspergillus transformation (kindly provided by Pat Kennedy and Dr. Larry Yager, Temple University, Philadelphia). One of the BamHI subclones, p/W17, contained a $5.3-\mathrm{kb}$ insert that complemented the $f l b D$ mutation at a high frequency. The insert from p/W17 was divided into two fragments: a $3.2-\mathrm{kb}$ BamHI-KpnI fragment and a 2.1-kb KpnI fragment, and each of the fragments was inserted into pPK1 to give pJW18 and p/W20, respectively. pJW18 complemented the $f l b D$ gene at high frequency, whereas p/W20 did not complement the mutation at all, indicating that the entire $f l b D$ gene was probably contained in the $3.2-\mathrm{kb}$ insert of pJW18.

The $f l b D$ disruption plasmid pJW30 was constructed by inserting a 4.1-kb XhoI $\operatorname{trpC}$ fragment from pTAll into an Xhol site 410 bp downstream of the putative FlbD translation start site. The $a l c A(p):: f l b D$ fusion plasmid was made by inserting the 1.5-kb KpnI-EcoRI fragment of pJW25 downstream of the alcA promoter in pON2 to create pJW26. The $2.0-\mathrm{kb}$ $a l c A(p):: f l b D$ fragment from pJW26 was then moved as an SstI-
KpnI fragment into the SstI and KpnI sites of pSH96 (S.T. Han and T.H. Adams, unpubl.). pSH96 is a Bluescript II KS(-) derivative in which the $\mathrm{fl}$ replication origin was removed by digestion with SspI and replaced with the 5' 2556 bp of $\operatorname{trpC}$ (Yelton 1984) that had been blunt-ended by a fill-in reaction using Klenow fragment.

\section{Acknowledgments}

This work was supported by National Institutes of Health grant GM45252 to T.H.A. The Fujix BAS2000 phosphorimaging system was made available through the Gene Technologies Laboratory at Texas A\&M University and National Science Foundation grant BIR-9217251. We thank Dr. Dan Ebbole, Dr. Paul Hardin, and Dr. Debby Siegele for critically evaluating the manuscript; Drs. Greg May and Bruce Miller for providing cDNA and cosmid libraries; and our colleagues in the laboratory for many helpful discussions.

The publication costs of this article were defrayed in part by payment of page charges. This article must therefore be hereby marked "advertisement" in accordance with 18 USC section 1734 solely to indicate this fact.

\section{References}

Adams, T.H., M.T. Boylan, and W.E. Timberlake. 1988. brlA is necessary and sufficient to direct conidiophore development in Aspergillus nidulans. Cell 54: 353-362.

Adams, T.H., H. Deising, and W.E. Timberlake. 1990. brlA requires both zinc fingers to induce development. Mol. Cell. Biol. 10: 1815-1827.

Adams, T.H., W.A. Hide, L.N. Yager, and B.N. Lee. 1992. Isola- 
tion of a gene required for programmed initiation of development by Aspergillus nidulans. Mol. Cell. Biol. 12: 38273833.

Altschul, S.F., W. Gish, W. Miller, W.W. Myers, and D.J. Lipman. 1990. Basic local alignment search tool. J. Mol. Biol. 215: 403-410.

Aramayo, R., T.H. Adams, and W.E. Timberlake. 1989. A large cluster of highly expressed genes is dispensable for growth and development in Aspergillus nidulans. Genetics 122: 6571.

Axelrod, D.E. 1972. Kinetics of differentiation of conidiophores and conidia by colonies of Aspergillus nidulans. J. Gen. Microbiol. 73: 181-184.

Axelrod, D.E., M. Gealt, and M. Pastushok. 1973. Gene control of developmental competence in Aspergillus nidulans. Dev. Biol. 34: 9-15.

Boylan, M.T., P.M. Mirabito, C.E. Willett, C.R. Zimmerman, and W.E. Timberlake. 1987. Isolation and physical characterization of three essential conidiation genes from Aspergil. lus nidulans. Mol. Cell. Biol. 7: 3113-3118.

Champe, S.P., M.B. Kurtz, L.N. Yager, N.J. Butnick, and D.E. Axelrod. 1981. In The fungal spore: Morphogenetic controls (ed. G. Turian and H.R. Hohl), pp. 63-91. Academic Press, New York.

Chang, Y.C. and W.E. Timberlake. 1992. Identification of Aspergillus brlA response elements (BREs) by genetic selection in yeast. Genetics 133: 29-38.

Clutterbuck, A.J. 1969. A mutational analysis of conidial development in Aspergillus nidulans. Genetics 63: 317-327.

- 1974. In Handbook of genetics (ed. R.C. King), pp. 447510. Plenum Publishing, New York.

Dietzel, C. and J. Kurjan. 1987. Pheromonal regulation and sequence of the Saccharomyces cerevisiae SST2 gene: A model for desensitization to pheromone. Mol. Cell. Biol. 7: 41694177.

Frampton, J., T.J. Gibson, S.A. Ness, G. Doderlein, and T. Graf. 1991. Proposed structure for the DNA-binding domain of the Myb oncoprotein based on model building and mutational analysis. Protein Eng. 4: 891-901.

Guehmann, S., G. Vorbrueggen, F. Kalkbrenner, and K. Moelling. 1992. Reduction of a conserved Cys is essential for Myb DNA-binding. Nucleic Acids Res. 9: 2279-2286.

Gwynne, D.I., F.P. Buxton, S. Sibley, R.W. Davies, R.A. Lockington, C. Scazzocchio, and H. Sealy-Lewis. 1987. Comparison of the cis-acting control regions of two coordinately controlled genes involved in ethanol utilization in Aspergillus nidulans. Gene 51: 205-216.

Han, S., J. Navarro, R.A. Greve, and T.H. Adams. 1993. Translational repression of $b r l A$ expression prevents premature development in Aspergillus. EMBO I. 12: 2449-2457.

Käfer, E. 1977. Meiotic and mitotic recombination in Aspergillus and its chromosomal aberrations. Adv. Genet. 19: 33131.

Katzen, A.L., T.B. Kornberg, and J.M. Bishop. 1985. Isolation of the proto-oncogene $c \cdot$ my $b$ from $D$. melanogaster. Cell 41: 449-456.

Law, D.J. and W.E. Timberlake. 1980. Developmental regulation of laccase levels in Aspergillus nidulans. I. Bacteriol. 144: $509-517$.

Lee, B.N. and T.H. Adams. 1994a. The Aspergillus nidulans flu $G$ gene is required for production of an extracellular developmental signal. Genes \& Dev. 8: 64l-651.

. 1994b. Overexpression of $f l b A$, an early regulator of $A s$ pergillus asexual sporulation leads to activation of $b r l A$ and premature initiation of development. Mol. Microbiol. 14: $323-334$.
Lockington, R.A., H.M. Sealy-Lewis, C. Scazzocchio, and R.W. Davies. 1985. Cloning and characterization of the ethanol utilization regulon in Aspergillus nidulans. Gene 33: 137149.

Lüscher, B. and R.N. Eisenman. 1990. New light on Myc and Myb. Part II. Myb. Genes \& Dev. 4: 2235-2241

Majello, B., L.C. Kenyon, and R. Dalla-Favera. 1986. Human $c-m y b$ proto-oncogene: Nucleotide sequence of cDNA and organization of the genomic locus. Proc. Natl. Acad. Sci. 83: 9636-9640.

Martinelli, S.D. and A.J. Clutterbuck. 1971. A quantitative survey of conidiation mutants in Aspergillus nidulans. J. Gen. Microbiol. 69: 261-268.

Miller, B., K. Miller, and W. Timberlake. 1985. Direct and indirect gene replacements in Aspergillus nidulans. Mol. Cell. Biol. 5: 1714-1721.

Morton, A.G. 1961. The induction of sporulation in mould fungi. Proc. R. Soc. Lond. B 153: 548-569.

Myrset, A.H., A. Bostad, N. Jamin, P. Lirsac, F. Toma, and O.S Gabrielsen. 1993. DNA and redox state induced conformational changes in the DNA-binding domain of Myb oncoprotein. $E M B O$ I. 12: 4625-4633.

Navarro-Bordonaba, J. and T.H. Adams. 1994. In Growth, differentiation, and sexuality (ed. J.G.H. Wessels and F. Meinhardt), pp. 333-349. Springer-Verlag, Berlin, Germany.

O'Donnel, K.L., A.H. Osmani, S.A. Osmani, and N.R. Morris. 1991. bimA encodes a member of the tetratricopeptide repeat family of proteins and is required for the completion of mitosis in Aspergillus nidulans. J. Cell Sci. 99: 711-719.

Ohi, R., D. McCollum, B. Hirani, G.D. Haese, X. Zhang, J.D. Burke, K. Turner, and K.L. Gould. 1994. The Schizosaccharomyces pombe $c d c 5^{+}$gene encodes an essential protein with homology to c-Myb. EMBO J. 13: 471-483.

Oppenheimer, D.G., P.L. Herman, S. Sivakumaran, J. Esch, and M.D. Marks. 1991. A myb gene required for leaf trichome differentiation in Arabidopsis is expressed in stipules. Cell 67: 483-493.

Pastushok, M. and D.E. Axelrod. 1976. Effect of glucose, ammonium and media maintenance on the time of conidiophore initiation by surface colonies of Aspergillus nidulans. I. Gen. Microbiol. 94: 221-224.

Paz-Ares, J., D. Ghosal, U. Wienand, P.A. Peterson, and H. Saedler. 1987. The regulatory $c 1$ locus of Zea mays encodes a protein with homology to myb proto-oncogene products and with structural similarity to transcriptional activators. EMBO I. 6: 3553-3558.

Peters, C.W.B., A.E. Sippel, M. Vingron, and K.H. Klempnauer. 1987. Drosophila and vertebrate myb proteins share two conserved regions, one on which funcions as a DNA-binding domain. EMBO /. 6: 3085-3090.

Pontecorvo, G., J.A. Roper, L.M. Hemmons, K.D. MacDonald, and A.W.J. Bufton. 1953. The genetics of Aspergillus nidulans. Adv. Genet. 5: 141-238.

Prade, R.A. and W.E. Timberlake. 1993. The Aspergillus nidulans brlA regulatory locus encodes two functionally redundant polypeptides that are individually required for conidiophore development. EMBO I. 12: 2439-2447.

Saikumar, P., R. Murali, and E.P. Reddy. 1990. Role of tryptophan repeats and flanking amino acids in Myb-DNA interactions. Proc. Natl. Acad. Sci. 87: 8452-8456.

Slamon, D.J., T.C. Boone, D.C. Murdock, D.E. Keith, M.F. Press, R.A. Larson, and L.M. Souza. 1986. Studies of the human $c-m y b$ gene and its product in human acute leukemias. Science 233: 347-351.

Stringer, M.A., R.A. Dean, T.C. Sewall, and W.E. Timberlake. 1991. Rodletless, a new Aspergillus developmental mutant 
induced by directed gene inactivation. Genes \& Dev. 5: 1161-1171.

Tice-Baldwin, K., G.R. Fink, and K.T. Arnt. 1989. BAS1 has an Myb motif and activates HIS4 transcription only in combination with BAS2. Science 246: 931-935.

Timberlake, W.E. 1980. Developmental gene regulation in Aspergillus nidulans. Dev. Biol. 78: 497-510.

Wieser, J., B.N. Lee, J.W. Fondon, and T.H. Adams. 1994. Genetic requirements for initiating asexual development in $A$ spergillus nidulans. Curr. Genet. 27: 62-69.

Yelton, M.M., J.E. Hamer, and W.E. Timberlake. 1984. Transformation of Aspergillus nidulans by using a trpC plasmid. Proc. Natl. Acad. Sci. 81: 1470-1474.

Yelton, M.M., W.E. Timberlake, and C.A.M.J.J. van den Hondel. 1985. A cosmid for selecting genes by complementation in Aspergillus nidulans: Selection of the developmentally regulated y $A$ locus. Proc. Natl. Acad. Sci. 82: 834-838. 


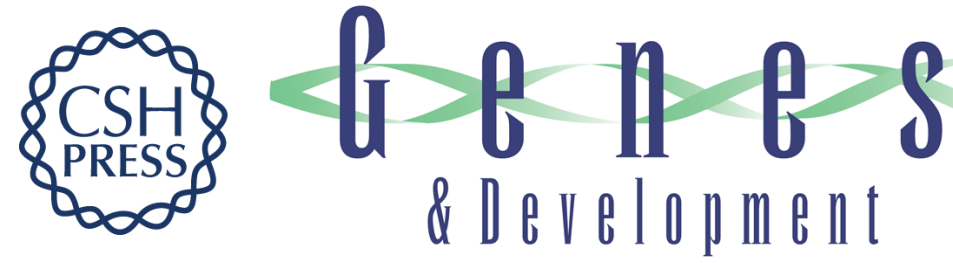

\section{flbD encodes a Myb-like DNA-binding protein that coordinates initiation of Aspergillus nidulans conidiophore development.}

$\mathrm{J}$ Wieser and T H Adams

Genes Dev. 1995, 9:

Access the most recent version at doi:10.1101/gad.9.4.491

References This article cites 43 articles, 18 of which can be accessed free at:

http://genesdev.cshlp.org/content/9/4/491.full.html\#ref-list-1

License

Email Alerting

Service

Receive free email alerts when new articles cite this article - sign up in the box at the top right corner of the article or click here.

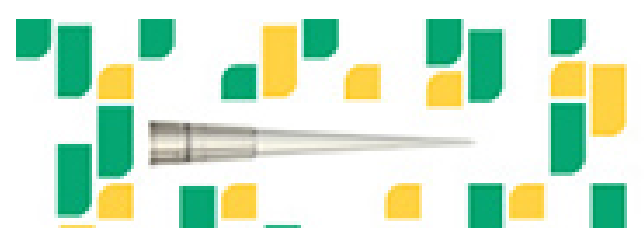

Focused on your science. 